\title{
Mycophenolate mofetil: safety and efficacy in the prophylaxis of acute kidney transplantation rejection
}

\author{
Pranav Dalal' \\ Monica Grafals ${ }^{2}$ \\ Darshika Chhabra² \\ Lorenzo Gallon² \\ 'Department of Medicine, Mount \\ Sinai Hospital, Chicago, USA; \\ ${ }^{2}$ Northwestern University-Feinberg \\ School of Medicine, Chicago, USA
}

\begin{abstract}
Mycophenolate mofetil (MMF), a prodrug of mycophenolic acid (MPA), is an inhibitor of inosine monophosphate dehydrogenase (IMPDH). It preferentially inhibits denovo pathway of guanosine nucleotide synthesis in T and B-lymphocytes and prevents their proliferation, thereby suppresses both cell mediated and humoral immune responses. Clinical trials in kidney transplant recipients have shown the efficacy of MMF in reducing the incidence and severity of acute rejection episodes. It also improves long term graft function as well as graft and patient survival in kidney transplant recipients. MMF is useful as a component of toxicity sparing regimens to reduce or avoid exposure of steroids or calcineurin inhibitor (CNI). Enteric-coated mycophenolate sodium (EC-MPS) can be used as an alternative immunosuppressive agent in kidney transplant recipients with efficacy and safety profile similar to MMF.
\end{abstract}

Keywords: mycophenolate mofetil, kidney transplantation, acute rejection, toxicity sparing

\section{Introduction}

Ongoing attempts to develop new immunosuppressive agents to achieve adequate immunosuppression with minimal toxicity have lead to discovery of several newer agents with different mechanism of actions. The introduction of mycophenolate mofetil in the mid-1990s has altered the management of immunosuppression in solid organ transplantation.

Mycophenolate mofetil (MMF) is an ester prodrug of mycophenolic acid (Figure 1). Mycophenolic acid (MPA)is a fermentation product of Penicillium brevicompactum and related fungi. ${ }^{1}$ It was discovered by Gosio in 1893 and was shown to have weak antibacterial activity. ${ }^{2}$ Its ability to inhibit inosine-5' -monophosphate dehydrogenase (IMPDH) was first identified in 1969. ${ }^{3}$ Initial studies with MMF in animal models of organ transplantation yielded encouraging results and led to the initiation of human trials. ${ }^{4,5}$ Sollinger et al conducted the first human trial of MMF in 1992 in kidney transplant recipients. ${ }^{6}$ Since then, MMF has been used in combination with other medications to prevent acute rejections, for rescue treatment in acute rejection episodes and as adjuvant to facilitate "sparing" of other immunosuppressive agents.

\section{Mechanism of action}

Two major pathways are involved in purine synthesis: the de novo pathway and the salvage pathway. MPA inhibits IMPDH, the rate-limiting enzyme in the de novo pathway of purine synthesis (Figure 2). By inhibiting IMPDH, MPA prevents formation of guanosine monophosphate (GMP). Cells depleted of GMP cannot synthesize guanine triphosphate (GTP) and deoxy guanine triphosphate (d-GTP), and therefore cannot replicate. Most mammalian cells are able to maintain GMP levels via the purine salvage pathway. MPA is 5-fold more potent as an inhibitor of the type II isoform of IMPDH, 


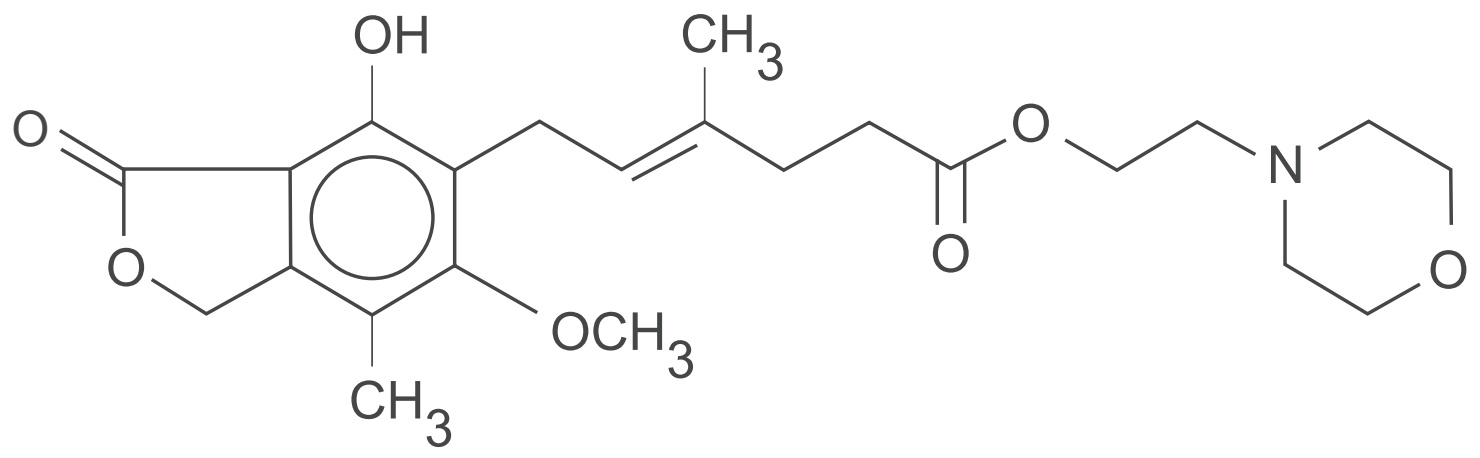

Figure I Chemical structure of mycophenolate mofetil (MMF) - the morpholinoethyl ester of mycophenolic acid (MPA) - mycophenolate.

which is expressed in activated $\mathrm{T}$ and $\mathrm{B}$ lymphocytes, than of the type I isoform, which is expressed in most cell types. ${ }^{7}$ Due to the expression of the more susceptible form of IMPDH, MPA preferentially inhibits the de novo guanosine nucleotide synthesis in lymphocytes. Monocytes are also affected by therapeutic doses of MPA, as it significantly decreases guanosine triphosphate (GTP) pools in human peripheral blood monocytes but not in neutrophils. ${ }^{8}$ By preferential depletion of guanosine and deoxyguanosine nucleotides in $\mathrm{T}$ and $\mathrm{B}$ lymphocytes, MPA suppresses both cell mediated immune responses and antibody formation, major factors in both acute and chronic allograft rejection.

In addition to inhibition of DNA synthesis in lymphocytes, depletion of guanosine nucleotides suppresses the expression of several adhesion receptors including vascular cell adhesion molecule 1 (VCAM-1), E-selectin, and P-selectin on vascular endothelial cells. ${ }^{9}$ This interferes with the attachment of leukocytes to endothelial cells and prevents the recruitment of lymphocytes and monocytes to sites of inflammation. ${ }^{10,11}$ Suppression of mononuclear cell recruitment is another mechanism by which MMF decreases acute and chronic graft rejection.

MMF causes depletion of GTP and thereby depletion of tetrahydrobiopterin - a cofactor that limits the rate of inducible nitric oxide synthases (iNOS) activity, but not that of endothelial nitric oxide synthases (eNOS). Activation of iNOS is correlated with renal allograft rejection. Suppression of iNOS activity and NO production is presumably one of the mechanisms by which MMF prevents allograft rejection. ${ }^{12}$

\section{Pharmacokinetics}

Pharmacokinetics of MMF has been investigated in healthy volunteers and in renal allograft recipients. MPA is poorly absorbed, so the 2-morpholinoethyl ester, mycophenolate mofetil was developed to allow oral dosing. Following oral administration, MMF absorbs rapidly and completely and undergoes hepatic de-esterification to form MPA - an active immunosuppressant. Bioavaibility of MPA from MMF is about $94 \%$ and reaches peak plasma concentration about 2 hours after oral administration. ${ }^{13}$ MPA undergoes hepatic glucuronidation to form mycophenolic acid glucuronide (MPAG), which is pharmacologically inactive. MPAG is secreted into the bile and it is converted back to MPA by gut bacteria. MPA is then reabsorbed and via hepatic recirculation produces second peak between 8 and 12 hours. ${ }^{13} \mathrm{MMF}$ gets excreted in the urine as MPAG, accounting for $90 \%$ of the administered MMF dose. ${ }^{14}$ In renal transplant recipients during acute renal impairment in the early post-transplant period, the plasma MPA concentrations are comparable to patients without renal failure, whereas plasma MPAG concentrations are 2- to 3-fold higher. Renal failure or hemodialysis has no effect on plasma concentration of free MPA and no dosage adjustment is required for such patients. ${ }^{15}$

Concomitant administration of other immunosuppressive agents can influence pharmacokinetics of MPA. Cyclosporine (CSA) inhibits the biliary excretion of MPAG, thereby reduces the enterohepatic recirculation of MPAG. Tacrolimus (TAC) and sirolimus (SRL) do not interfere with biliary excretion of MPAG. So, the second MPA peak due to enterohepatic circulation is more pronounced in patients receiving TAC or SRL compared to CSA. However, higher MPAG concentration displaces MPA from binding sites and increases free MPA concentration, possibly similar to that seen with TAC or SRL based therapy. ${ }^{16-18}$ In the clinical setting, the level of MPA exposure correlates with the risk of developing acute rejection. MPA area under the curve (AUC) $<30 \mathrm{mg}$.h/L was associated with significant increased risk of acute allograft rejection and there was no additional reduction in the allograft rejection found for MPA AUC $>60 \mathrm{mg} . \mathrm{h} / \mathrm{L}$ in kidney transplant recipients on 
De novo Pathway

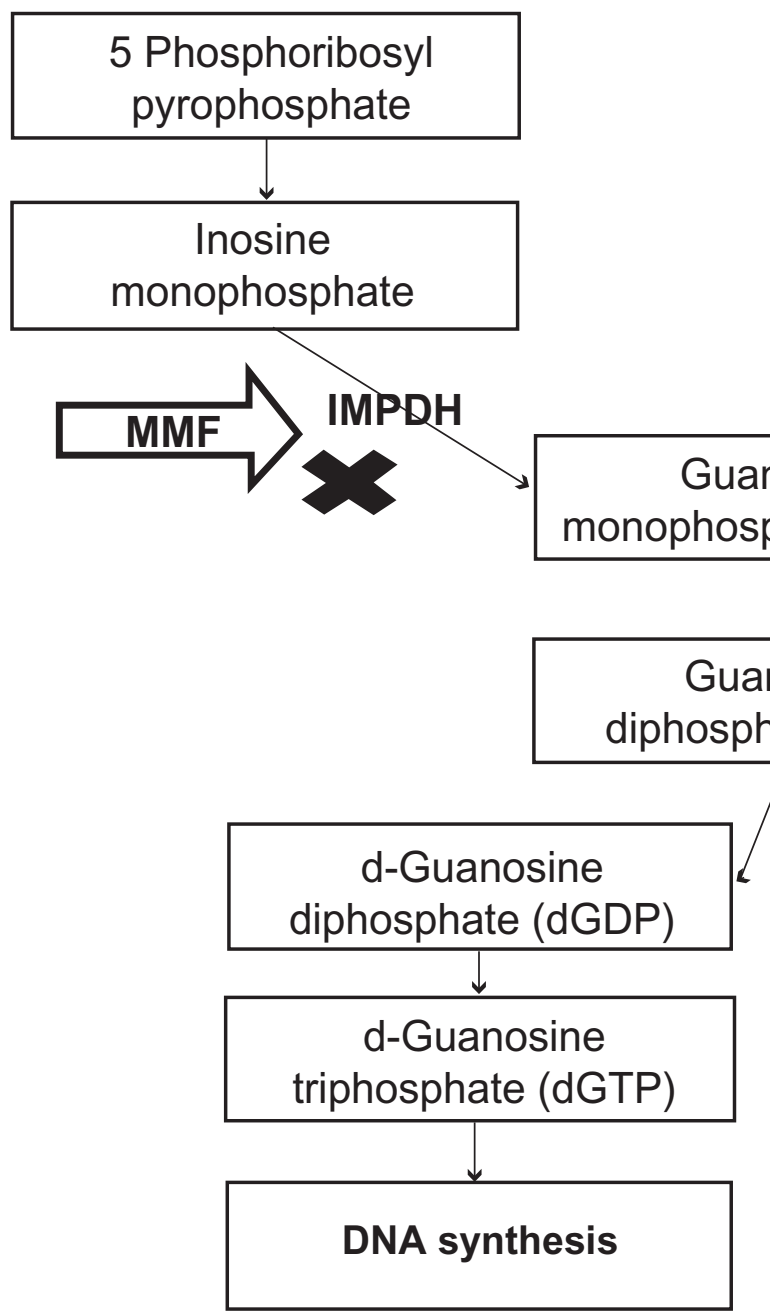

Salvage Pathway

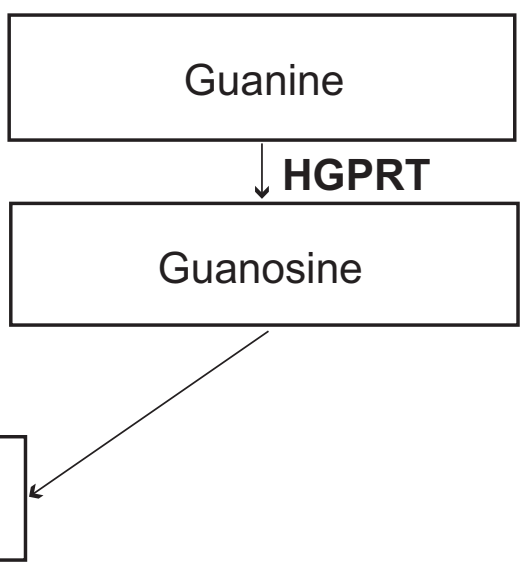

Figure 2 Mechanism of action - Inhibition of de novo pathway of purine synthesis by mycophenolate mofetil. Abbreviations: HGPRT, hypoxanthine-guanine phosphoribosyl; IMPDH, inosine monophosphate dehydrogenase.

conventional doses of CSA. ${ }^{19,20}$ The proposed therapeutic window of the MPA AUC ${ }_{0-12}$ is 30 to $60 \mathrm{mg} . \mathrm{h} / \mathrm{L}$. Currently, MPA therapeutic level monitoring is recommended only during early post-transplantation period for the patients at higher risk of developing acute allograft rejection. ${ }^{21}$ Usefulness of abbreviated 2-hour MPA AUC measurement to predict MPA AUC $_{12 \text { hour }}$ has been suggested by some studies. ${ }^{22,23}$ However, this abbreviated sampling strategy require strict adherence to the blood sample collection time and it can miss MPA enterohepatic recirculation, which is especially important in patients on simultaneous TAC or SRL therapy. ${ }^{15}$

\section{Prevention of acute renal allograft rejection}

Acute allograft rejection usually occurs within the first 3 to 6 months post transplantation, being one of the most significant problems in transplant recipients. Acute rejection is not only the principal cause of early graft loss, but it also increases the risk of late graft loss by predisposing to irreversible chronic rejection. MMF has been shown to prevent acute graft rejection following renal transplantation in animal experiments. ${ }^{4,5}$

The evidence of safety and efficacy of MMF in the prevention of acute renal allograft rejection from three large, randomized, multicenter, human clinical trials was instrumental in MMF being approved by the US Food and Drug Administration (FDA) and European agencies. All three studies (Table 1) had similar design, using MMF 2 g/day (1000 mg bid) or $3 \mathrm{~g} /$ day (1500 mg bid) plus CSA and corticosteroids as an immunosuppressive regimen, with or without induction therapy. ${ }^{24-26}$ The US and Tricontinental study groups compared MMF with azathioprine (AZA), while the European study 
group compared MMF with placebo. The primary efficacy endpoint was biopsy-proven acute rejection (BPAR) or treatment failure (defined as allograft loss, patient withdrawal or death) during the first 6 months after transplantation.

US Renal Transplant Mycophenolate Mofetil Study Group randomized 499 patients to either MMF (2 g/d or $3 \mathrm{~g} / \mathrm{d}$ ) or AZA 1 to $2 \mathrm{mg} / \mathrm{kg} / \mathrm{d}$. Cyclosporine, corticosteroids and antithymocyte globulin were administered as part of a quadruple sequential induction protocol. The incidence of first BPAR or treatment failure was significantly reduced in the MMF $2 \mathrm{~g} / \mathrm{d}$ group $(31.1 \%, \mathrm{p}=0.0015)$ and in the MMF $3 \mathrm{~g} / \mathrm{d}$ group $(31.3 \%, \mathrm{p}=0.0021)$, compared to $47.6 \%$ in the AZA group. First episode of BPAR alone occurred in $38 \%$ of patients who received AZA compared with $19.8 \%$ and $17.5 \%$ of patients who received MMF $2 \mathrm{~g} / \mathrm{d}$ and $3 \mathrm{~g} / \mathrm{d}$, respectively. Time to first BPAR episode or treatment failure was significantly longer for MMF $2 \mathrm{~g} / \mathrm{d}$ vs AZA $(\mathrm{p}=0.0036)$ and MMF $3 \mathrm{~g} / \mathrm{d}$ vs AZA $(\mathrm{p}=0.0006) .{ }^{26}$

In the European Mycophenolate Mofetil Cooperative Study Group, 491 patients were randomized to either placebo, MMF $2 \mathrm{~g} / \mathrm{d}$ or MMF $3 \mathrm{~g} / \mathrm{d}$. Patients received CSA and corticosteroids as part of maintenance immunosuppression. However, no induction therapy was given. At 6 months, the incidence of BPAR or treatment failure was 56.0\%, 30.3\% and $38.8 \%$ in placebo, MMF $2 \mathrm{~g} / \mathrm{d}$ and MMF $3 \mathrm{~g} / \mathrm{d}$ groups respectively ( $\mathrm{p}<0.001$ for both $\mathrm{MMF}$ groups, compared to placebo). The corresponding percentages for BPAR were $46.4 \%, 17.0 \%$ and $13.8 \%$ respectively. ${ }^{24}$

The Tricontinental Mycophenolate Mofetil Renal Transplantation Study Group compared the efficacy and safety of MMF with AZA, in patients receiving a first or second cadaveric renal graft in a prospective double blind, randomized trial. No induction therapy was given. BPAR or treatment failure occurred in $50 \%$ of patients in the AZA group by 6 months after transplantation, compared with $38.2 \%$ in the MMF $2 \mathrm{~g} / \mathrm{d}$ group $(\mathrm{p}=0.0287)$ and $34.8 \%$ in the MMF $3 \mathrm{~g} / \mathrm{d}$ group $(\mathrm{p}=0.0045)$. The incidence of BPAR was $19.7 \%$ in the MMF $2 \mathrm{~g} / \mathrm{d}$ group, $15.9 \%$ in the MMF $3 \mathrm{~g} / \mathrm{d}$ group and $35.5 \%$ in the AZA group. At the end of 1 year after transplantation, the graft survival in the MMF groups was superior to that in the AZA group, although this difference was not statistically significant. ${ }^{25}$

Efficacy data analysis from the three trials mentioned above, comprising 1493 patients, showed superiority of MMF $2 \mathrm{~g} / \mathrm{d}$ and MMF $3 \mathrm{~g} / \mathrm{d}$ over AZA or placebo in preventing biopsy proven acute allograft rejection at 12 months post transplantation. The rejection episodes were less severe in the MMF group. Also, the need for anti lymphocyte globulin and the number of full courses of steroids required to treat rejection episodes were reduced by more than $60 \%$ in MMF group. There was an absolute reduction in the incidence of graft loss due to acute rejection in the MMF group compared to the AZA or the placebo group. Although, MMF treatment reduced the incidence and severity of acute rejection episodes, there was no significant difference in patient survival and graft survival at the end of 1 year. ${ }^{27}$

All three trials were done using the old formulation of CSA. Remuzzi et al (MYSS trial) used the micro-emulsion preparation of CSA (Neoral) in patients receiving their first kidney transplant from cadaveric donors. ${ }^{28}$ During phase A, all patients received either MMF $2 \mathrm{~g} / \mathrm{d}$ or AZA $(100 \mathrm{mg} / \mathrm{d}$ if bodyweight $<75 \mathrm{~kg}, 150 \mathrm{mg} / \mathrm{d}$ if $>75 \mathrm{~kg}$ ) and steroids for immunosuppression for 6 months. At the end of 6 months, patients with less than 2 episodes of acute rejection, no episodes of steroid resistant rejection, stable serum creatinine level and urinary protein excretion less than 1 $\mathrm{g} / \mathrm{d}$ entered phase B. During phase B, steroids were gradually tapered and discontinued over 90 days in both groups. At the end of 6 months (phase A) and 21 months (phase B), the incidence of BPAR was similar in the MMF and AZA groups. Graft losses due to refractory rejections and adverse events were also comparable in both groups. Cost of MMF therapy was 15 times higher than AZA therapy. In this study, investigators monitored and tried to maintain trough CSA levels between 150 and $250 \mu \mathrm{g} / \mathrm{L}$, which was achieved in most patients. This could probably explain low rejection rates in both groups. ${ }^{28}$

Table I Summary table of MMF pivotal trials

\begin{tabular}{lllll}
\hline Trial & MMF group & Control group & Induction & BPAR at 6 months \\
\hline US Renal Transplantation Study ${ }^{26}$ & MMF/CSA/CS & AZA/CSA/CS & ATG & MMF better \\
European Study ${ }^{24}$ & MMF/CSA/CS & Placebo/CSA/CS & No & MMF better \\
Tricontinental Study 25 & MMF/CSA/CS & AZA/CSA/CS & No & MMF better \\
MYSS Study & MMF/Neoral/CS & AZA/Neoral/CS & No & No difference \\
\hline
\end{tabular}

Abbreviations: MMF, mycophenolate mofetil; AZA, azathioprine; CS, corticosteroids; CSA, cyclosporine; ATG, antithymocyte globulin; Neoral, microemusion cyclosporine; BPAR, biopsy proven acute rejection. 
At the end of 72 months of follow up of patients in the MYSS trial, investigators found that the incidences of graft loss $(6.8 \%$ vs $6.1 \%, p=0.82)$, late rejections $(>6$ mo after transplantation, $25.3 \%$ vs $21.2 \%$; $=0.53$ ), mean glomerular filtration rate (GFR) and adverse events were similar in the AZA $(n=124)$ and the MMF $(n=124)$ groups, respectively. Since both drugs, AZA and MMF have similar risk/benefit profiles, authors of this study recommended routine use of AZA over MMF in combination with micro-emulsion preparation of CSA to save on the cost of immunosuppressive therapy. ${ }^{29}$

\section{Long term allograft survival}

In the above studies, MMF was comparable to or better than AZA in reducing the episodes of acute allograft rejections at 1 year post transplantation. The Tricontinental study group reported the impact of long-term MMF use on allograft survival. At the end of 3 years, analysis of the Tricontinental study showed similar graft survival with AZA and both strengths of MMF, but graft loss due to acute rejection was reduced in the MMF $2 \mathrm{~g} / \mathrm{d}$ and $3 \mathrm{~g} / \mathrm{d}$ groups (5.8\% and $3 \%$, respectively), compared to $9.9 \%$ in the AZA group. Of patients who experienced a BPAR episode within 6 months of transplantation, $31.5 \%$ lost their graft by the end of 3 years. In contrast, only $6.6 \%$ of patients who had no early acute rejection lost their graft by the end of the 3-year study period. Adding MMF to the standard immunosuppressive therapy and thereby reducing the incidence of acute rejection may also result in an improvement in long-term graft and patient survival. ${ }^{30}$

Retrospective analysis of data collected between 1988 and 1997 of 66,774 renal transplant recipients in the US registry, confirmed protective effect of MMF on long term allograft survival. Acute rejection was the strongest risk factor for late graft loss with risk ratio of 2.41 ( $p<0.001)$. MMF therapy was associated with a decrease in the incidence of acute rejection, compared to AZA. In a multivariate analysis, after controlling for acute rejection, MMF therapy reduced the relative risk for development of chronic allograft failure (CAF) by 27\% (risk ratio [RR] 0.73, p < 0.001) compared to AZA therapy. This analysis confirmed that MMF therapy was an independent protective factor in preventing $\mathrm{CAF}$, not related to the decrease in acute allograft rejection. The authors suggested that the independent protective effect of MMF was possibly related to prevention of chronic allograft arteriopathy by decreasing vascular intimal hyperplasia. ${ }^{31}$

Analysis of data from the US renal system of 49,666 primary renal transplant recipients evaluated the effect of long-term continuous MMF or AZA use on renal allograft function. The results showed that the continuous use of MMF had a protective effect against declining renal function, as measured by the slope of reciprocal creatinine, compared to continuous AZA use $(\mathrm{p}<0.001) .{ }^{32} \mathrm{MMF}$ was associated with a $65 \%$ reduced risk of late acute rejection compared with AZA ( $p<0.001)$. The incidence of acute rejection episodes at 2 and 3 years post transplantation was significantly lower in the MMF group ( $0.9 \%$ at 2 years, $1.1 \%$ at 3 years) than in the AZA group (6.1\% at 2 years, $9.3 \%$ at 3 years). ${ }^{33}$

\section{Rescue therapy for acute renal allograft rejection}

Trials have looked at the efficacy of MMF as treatment for acute refractory rejection episodes, both as an alternative and as an adjunct to high-dose corticosteroids. The MMF Renal Refractory Rejection Study Group, in an open label randomized multicenter trial, compared the efficacy and safety of MMF with high dose intravenous (IV) steroids for the treatment of refractory, acute cellular rejection in recipients of first or second cadaveric or living-donor renal allografts. A total of 150 patients were randomized to receive either high-dose IV steroids for 5 days followed by a 5-day taper to $20 \mathrm{mg} / \mathrm{d}(\mathrm{n}=77)$ or oral MMF $1.5 \mathrm{~g}$ twice daily $(\mathrm{n}=73)$. Patients treated with high dose steroids were maintained on AZA. All patients received maintenance CSA and steroid therapy. MMF reduced graft loss and death by $45 \%$ compared to IV steroid group at the end of 6 months. The number of patients requiring full courses of IV steroids for subsequent rejection episodes were similar in both groups but patients who received full courses of anti lymphocyte globulin were more than double in IV steroid group $(n=18)$ compared to the MMF group $(n=8){ }^{34}$

A two-phase trial evaluated MMF as an adjunctive therapy in 221 patients with acute rejection episodes. In phase 1, patients with acute rejection between 7 days and 6 months post transplantation were randomized to receive IV steroids combined with either MMF (1.5 g twice daily) or AZA. At the end of 1 year, incidence of recurrent or persistent rejection or treatment failure was significantly lower in the MMF group compared to the AZA group (29.2\% vs $51.9 \%$, respectively; $\mathrm{p}=0.0006$ ). Patients in the AZA group were also more likely to require at least one course of antithymocyte globulin. ${ }^{35}$

In summary, MMF was found to be more effective as a rescue therapy in acute renal allograft rejection treatment as compared to AZA or high dose IV steroid treatment. Treatment with MMF also reduced the episodes of subsequent rejections and graft loss due to acute rejection. 


\section{Toxicity-sparing protocols}

Use of triple drug maintenance regimens including - a calcineurin inhibitor, steroids and MMF, have reduced the incidence of acute rejection in renal transplant recipients. ${ }^{36}$ However, the long-term use of steroids and CNIs is associated with numerous potentially toxic effects that can impact overall graft or patient survival. Recent studies have evaluated MMF as part of different immunosuppressive maintenance regimens to facilitate withdrawal or avoidance of steroids and CNIs in renal transplant recipients.

\section{Calcineurin inhibitors-sparing regimens}

CNIs have been useful in reducing the incidence acute allograft rejections during the early post-transplantation period. ${ }^{37,38}$ Nephrotoxicities like renal vasoconstriction, reduced glomerular filtration rate, arterial hypertension and interstitial fibrosis, can develop in both native and transplanted kidneys with prolonged use of CNIs. Other adverse effects of CNIs use include hyperlipidemia, neurotoxicity and impaired glucose metabolism. ${ }^{39-41}$ These can ultimately lead to chronic allograft dysfunction and poor patient and/or graft survival. Since MMF has emerged as highly effective immunosuppressive agent with no known nephrotoxicity, attempts have been made to develop protocols to minimize CNIs exposure by dosage reduction, withdrawal or complete avoidance, to improve long term allograft survival.

Initially, studies were done to withdraw CSA in renal transplant recipients with stable renal function at 12 months post transplantation. In a prospective study, 64 stable renal transplant recipients on CSA and prednisone were randomized for conversion of CSA to $2 \mathrm{mg} / \mathrm{kg} / \mathrm{d}$ AZA $(\mathrm{n}=30)$ or $1 \mathrm{~g}$ of MMF twice daily $(\mathrm{n}=34)$. All patients remained on low dose steroids. At the end of four months after conversion, the incidence of acute rejection was lower in the MMF group (4/34) compared to the AZA group $(11 / 30)(p=0.04) .{ }^{42}$ In two other studies, the incidence of acute rejection using MMF and steroids to withdraw CSA at 12 months after transplantation was similar to the above study (10.6\% and $11.8 \%))^{43,44}$ A significant number of patients in the CSA withdrawal group had improved renal function in all three studies. ${ }^{42-44}$

While the above studies established safety and efficacy of MMF in CNI withdrawal in patients with stable renal function, in the "Creeping Creatinine Study", Dudley et al attempted to withdraw CSA in patients with deteriorating renal function at least 6 months post transplantation. These patients were randomized to either continue CSA or to add MMF with gradual withdrawal of CSA over a 6-week period. At 6 month follow up, patients in CSA withdrawal/MMF group had stabilization or improvement in renal function without increase in acute allograft rejection. ${ }^{45}$

Complete avoidance of CNIs was attempted using MMF based immunosuppression in some trials recently. In most of the studies, some form of induction therapy was used to avoid early post-transplantation rejection. ${ }^{46-54}$ In a randomized study comparing MMF/SRL/steroids to MMF/TAC/steroids, Larson et al did not find any difference in renal function at 12 months post transplantation. The incidence of acute rejection was $10 \%$ in the TAC group and $13 \%$ in the SRL group $(\mathrm{p}=0.58)$. Patient survival and graft survival were also comparable in both groups.$^{55}$ Flechner et al published 5-year outcome data from a randomized prospective trial in primary adult renal allograft recipients, comparing MMF/SRL/steroids $(n=31)$ to MMF/CSA/steroids $(n=30)$. At 5 years both groups had comparable acute rejection rates $(12.9 \%$ vs $23.3 \%, p=0.22)$ and patient survival $(87.1 \%$ vs $90 \%, \mathrm{p}=0.681)$. Although unadjusted patient survival was similar, SRL/MMF based CNI-free patients had longer death censored graft survival ( $96.4 \%$ vs $76.7 \%, p=0.0265$ ), higher glomerular filtration rate ( $66.7 \mathrm{vs} 50.7 \mathrm{cc} / \mathrm{min}, \mathrm{p}=0.0075$ ), and fewer graft losses from chronic allograft nephropathy. ${ }^{56}$

Investigators evaluated efficacy and safety of four different MMF based immunosuppressive regimens in the Elite-Symphony study. They randomized 1645 renal transplant recipients to receive standard-dose CSA, MMF and corticosteroids, or daclizumab induction, MMF and corticosteroids in combination with low-dose CSA, or lowdose TAC, or low-dose SRL. At the end of 12 months post transplantation, the rate of BPAR was significantly lower in MMF/TAC group compared to the other three groups. The mean calculated GFR and overall graft survival were also better in the MMF/TAC group. ${ }^{57}$

Srinivas et al analyzed the data of solitary kidney transplant recipients reported to the Scientific Registry of Renal Transplant Recipients (2000-2005) to compare outcomes of different immunosuppressive regimens. MMF/SRL combination was associated with higher risk of acute rejection at the end of 6 months post transplantation compared to MMF/TAC ( $p<0.01)$. Kaplan-Meier analysis showed inferior overall graft and patient survival with MMF/SRL compared to MMF/TAC and MMF/CSA at the end of 3 years post transplantation..$^{58}$

\section{Steroid-sparing regimens}

Steroid therapy in transplant recipients is associated with the development of several side effects like hypertension, weight gain, poor glucose tolerance, dyslipidemia, osteoporosis 
and hip fracture, avascular necrosis of joints and sepsis. ${ }^{59}$ However, withdrawal of steroids can precipitate acute rejection episodes in renal transplant recipients. Before the era of MMF, trials with late steroid withdrawal (>3 months post transplantation) were associated with a significant increase in acute allograft rejection and late graft loss. ${ }^{60,61}$ In a prospective randomized steroid withdrawal trial using AZA and CSA as maintenance therapy, 2-year graft survival was $78 \%$ in early steroid withdrawal group (at 2 weeks) vs $86 \%$ in later steroid withdrawal group ( $>6$ months post transplantation). Withdrawal of steroids was successful in only 13 of 42 patients (41\%) in the early withdrawal group and 59 of 75 patients $(79 \%)$ in the late withdrawal group. ${ }^{62}$ Meta-analysis of trials of late steroid withdrawal in renal transplant recipients maintained on CSA with/without AZA also reported higher risk of acute rejection and graft failure after steroid withdrawal. ${ }^{63,64}$ With the addition of newer immunosuppressive drugs like MMF, TAC, SRL and agents for induction therapy, safety of steroid withdrawal has improved. MMF has been used as part of steroid sparing immunosuppressive regimens in combination with various other agents.

In two different trials, Squifflet et al and Vantenterghem et al used MMF/TAC and MMF/CSA respectively, to withdraw patients from steroids at 3 months post transplantation. At the end of the study period, there was no significant difference noted in the incidence of acute allograft rejection or graft/patient survival between the two groups. The steroid withdrawal group had lower cholesterol and triglyceride levels and blood pressure was better controlled. ${ }^{65,66}$

More recently investigators have tried restricting steroid use to the first few critical days post-transplantation. Matas et al published data from a pilot trial withdrawing steroids at day 5 post transplantation in 51 living donor recipients. Antithymocyte globulin was given for induction and MMF/CSA were given as maintenance regimen. At the end of 1 year, rejection-free graft survival and patient survival were similar between the groups. ${ }^{67}$ In 3-year follow up data on 349 transplant recipients using the same rapid steroid withdrawal regimen, they reported $94 \%$ and $92 \%$ acute rejection free graft survival at the end of 1 year and 3 years respectively. The overall patient survival and graft survival were excellent compared to historic controls. ${ }^{68}$

Vincenti et al randomized patients to early steroid withdrawal or steroid maintenance and found no significant differences in acute rejection episodes or patient/graft survival. Patients also received basiliximab induction and $\mathrm{MMF} /$ CSA maintenance therapy ${ }^{69}$ In another study by the same group, de novo kidney transplant patients were randomized to receive no steroids $(n=112)$, steroids up to day $7(n=115)$, or standard steroids $(n=109)$ with CSA microemulsion, entericcoated mycophenolate sodium (EC-MPS) and basiliximab. The 12-month incidence of BPAR, graft loss or death was significantly lower in the standard steroid group compared to the two other groups. Renal function was similar in all three groups. The de novo use of anti-diabetic and lipid lowering medication was lower in one or both steroid minimization groups compared to standard steroids group. ${ }^{70}$

In another trial, 186 patients were randomized to steroid withdrawal at day 3 in conjunction with daclizumab induction and $\mathrm{MMF} / \mathrm{CSA}$ maintenance compared to 178 patients to steroid withdrawal at 4 months with MMF/CSA maintenance without induction therapy. At the end of 1 year, similar percentages of patients were steroid free with functioning graft in both groups and the rate of acute rejection was $15 \%$ in early withdrawal group vs $14 \%$ in late withdrawal group. ${ }^{71}$

In a single center, retrospective sequential analysis of 212 renal transplant recipients with median follow up of 5 years by Gallon et al there was no significant difference between rate and severity of acute rejection episodes, graft survival, patient survival and decline of renal function between chronic steroid maintenance group $(\mathrm{n}=96)$ and rapid steroid elimination group $(n=116)$. All patients also received induction with IL-2 receptor antagonist and maintenance immunosuppression with MMF and TAC. Patients in the chronic steroid group had higher incidence of hyperlipidemia and post transplantation diabetes compared to the rapid steroid elimination group. ${ }^{72}$

A meta-analysis of 6 randomized steroid withdrawal trials, while receiving MMF based immunosuppression showed that the risk of acute rejection episodes after steroid elimination was 2.28 times higher than that observed in patients maintained on steroids, but this did not affect graft survival at medium term follow up. The reduction in total cholesterol level was significant in the steroid withdrawal group. ${ }^{73}$

Although, the benefits of eliminating steroid related side effects are obvious, there is ongoing debate about the long-term safety of steroid-free maintenance immunosuppression protocols. Current studies on steroid free, MMF based immunosuppression maintenance protocols in kidney transplantation recipients, using $\mathrm{T}$ cell depleting induction therapy will provide more definite answers in near future.

\section{Safety and tolerability}

The data derived from three pivotal trials established the safety and tolerability of MMF in adult renal transplantation 
recipients. MMF was generally well tolerated in most studies. The commonly reported adverse effects were gastrointestinal (GI) tract related including nausea, vomiting, diarrhea and abdominal pain. While the frequency of nausea was similar in the AZA, MMF $2 \mathrm{~g} / \mathrm{d}$ and MMF $3 \mathrm{~g} / \mathrm{d}$ groups, the frequency of vomiting, diarrhea and abdominal pain were higher with the MMF $2 \mathrm{~g} / \mathrm{d}$ and MMF $3 \mathrm{~g} / \mathrm{d}$ groups compared to the AZA group. Most symptoms usually resolved with dose reduction, interruption or withdrawal of MMF. ${ }^{24-26}$ Various studies in renal transplantation patients had $20 \%$ to $40 \%$ of patients requiring either dose reduction or withdrawal of MMF due to GI side effects. ${ }^{74-76}$ The impact of dose change and clinical outcome has been reported in several studies. In one study, patients with MMF dose changes during the first year of transplantation had higher incidence of acute allograft rejection and significant decrease in 3-year death censored allograft survival compared to those who did not. ${ }^{75} \mathrm{Knoll} \mathrm{et} \mathrm{al}$ found an increase in relative risk of acute rejection by $4 \%$ for every week that MMF dose was reduced below the full dose $(p=0.02) .{ }^{77}$ In an analysis of the US Renal Data System (USRDS) database of cadaveric renal transplant recipients between 1995 to 1998, who were treated with MMF and had a functioning graft at 1 year post transplantation, the incidence of discontinuation of MMF was higher in patients with GI side effects, adding an additional cost of US\$4500 to 8000 to the second year after transplantation. ${ }^{74}$

All three pivotal trials reported higher incidence of invasive cytomegalovirus (CMV) infections with MMF compared to AZA, especially in patients receiving higher dose of MMF $3 \mathrm{~g} / \mathrm{d}$. The incidence of CMV infection was also higher with MMF $2 \mathrm{~g} / \mathrm{d}$ than AZA at 6 months, 1 year and 3 years post transplantation, but was not statistically significant. CMV infection incidence was not significantly different between MMF $2 \mathrm{~g} / \mathrm{d}$ and MMF $3 \mathrm{~g} / \mathrm{d}$ groups. The increased incidence was possibly related to higher endoscopic surveillance as a part of work up for GI symptoms and the absence of standards of cytomegalovirus prophylaxis. ${ }^{24-26}$

At 1 and 3 years post transplantation, the incidence of leukopenia was significantly higher in the MMF $3 \mathrm{~g} / \mathrm{d}$ group compared to the AZA group (RR 1.13, 1.37, p $<0.05$ respectively). In contrast, the incidence of leukopenia was lower with MMF 2/d than AZA at 6 months, 1 year and 3 years post transplantation, but these differences were not statistically significant. There was no significant difference in the occurrence of anemia in all three groups at 1 year and 3 years post transplantation. Although the incidence of thrombocytopenia was lower with MMF $3 \mathrm{~g} / \mathrm{d}$ compared to AZA at 1 year post transplantation, there was no significant difference found between MMF $2 \mathrm{~g} / \mathrm{d}, \mathrm{MMF} 3 \mathrm{~g} / \mathrm{d}$ and AZA at the end of 3 years. ${ }^{78}$

The overall skin malignancy incidence was similar for MMF $3 \mathrm{~g} / \mathrm{d}$ and AZA groups at 3 years post transplantation. There was no statistical difference in skin malignancy incidence between MMF $2 \mathrm{~g} / \mathrm{d}$ and AZA at 1 year and 3 years post transplantation. ${ }^{78}$

\section{Enteric coated mycophenolate sodium (EC-MPS)}

Gastrointestinal complaints associated with MMF therapy have shown to impair physical, social and psychological functioning in renal transplant recipients. ${ }^{79}$ Dose reduction, interruption or discontinuation of MMF in patients with GI complaints increases the risk of acute rejection or graft loss. ${ }^{74,76,77,80}$ Enteric-coated mycophenolate sodium was developed to avoid GI side effects and to improve patient compliance, without compromising drug dose or efficacy. EC-MPS $720 \mathrm{mg}$ tablet and MMF $1000 \mathrm{mg}$ tablet provides similar MPA maximal plasma concentration and MPA exposure. $^{81}$

Therapeutic equivalence of MMF (1000 mg bid) and EC-MPS ( $720 \mathrm{mg}$ bid) was assessed in a randomized, double blind study involving 423 de novo kidney transplant recipients. Efficacy failure and safety profile were assessed at 6 months and at 12 months. The incidences of BPAR, graft loss, death and loss to follow up were similar in both groups at 6- and 12-month periods. Among those with BPAR, the incidence of severe acute rejection was lower with EC-MPS compared to MMF (9.8\% with MMF and 2.1\% with EC-MPS; $\mathrm{p}=$ not significant). The safety profile and incidence of GI adverse events were comparable for both groups. Within 12 months, $19.5 \%$ of MMF patients and 15\% of EC-MPS patients required dose changes for GI adverse events. This study revealed that MMF $1000 \mathrm{mg}$ bid and EC-MPS $720 \mathrm{mg}$ bid are therapeutically equivalent with similar safety profiles in de novo renal transplant patients. ${ }^{82}$

In an open label extension of the above study, 247 patients received EC-MPS $720 \mathrm{mg}$ bid during the 12-month to 36-month post-transplantation period. The type and severity of adverse events were comparable between the newly exposed and long term EC-MPS patients during first 24-month extension. The incidence of BPAR was 5\% (6 patients) in newly exposed group and 3\% (4 patients) in long term EC-MPS group. The data from this study were compared with data of MMF-treated patients taking part in two other studies comparing MMF versus everolimus. In cross study comparisons, the incidence of efficacy events 
and safety profile, including GI side effects, infections and malignancies were comparable between the two groups. ${ }^{83}$

In a pivotal, phase III, randomized, multicenter, doubleblind, double-dummy, parallel group study investigated safety and efficacy of EC-MPS in stable renal transplant recipients. The stable renal transplant recipients on MMF $1000 \mathrm{mg}$ bid maintenance therapy with concomitant CSA (with/without steroids) were either continued on MMF (1000 mg bid) or switched to EC-MPS (720 mg bid). GI adverse events were comparable in both groups at 3 and 6 months, with a trend favoring EC-MPS. Overall incidence of infections was similar in both groups, but there were fewer serious infections in the EC-MPS group ( $p<0.05)$. The combined incidence of BPAR, graft loss, death or loss to follow up was similar in both groups (EC-MPS 7.5\% vs MMF $12.3 \%, \mathrm{p}<0.05) .{ }^{84}$ At 12 months, in an open label extension, 130 patients initially randomized to MMF were converted to EC-MPS (newly exposed) and 130 patients initially randomized to EC-MPS were continued on EC-MPS (EC-MPS long term). At the end of 12-month extension period, incidence and type of adverse events, including GI side effects and malignancy were similar in both groups. Mean serum creatinine level was also similar in both groups. ${ }^{85}$

In two pooled analysis; the first about the use of EC-MPS in de novo renal transplant patients including three multicenter studies $(n=456)$ and the second about conversion of MMF to EC-MPS in stable renal transplant patients also including three multicenter studies $(n=564)$, EC-MPS was well tolerated with comparable safety and efficacy profile to MMF. ${ }^{86,87}$

These studies revealed that EC-MPS has efficacy and safety profile similar to MMF in de novo renal transplant patients. Also, stable renal transplant can be safely converted from MMF to EC-MPS maintenance therapy and it can be given for long time.

In conclusion, MMF has become a mainstay of immunosuppressive treatment in kidney transplantation recipients, reducing acute rejection episodes in the short term and improving allograft and patient survival in the long term. MMF in combination with new immunosuppressive agents can be safely utilized to offer CNI or steroid sparing regimens to reduce side effects. Although, MMF is usually well tolerated with benign side effect profile, gastrointestinal adverse effects are a major concern. EC-MPS has an identical safety and efficacy profile to MMF and can be used as an alternative immunosuppressive agent in de novo and stable kidney transplantation recipients.

\section{Disclosures}

There was no financial support received to carry out the above study. There has been no commercial or proprietary interest in any drug, device, or equipment mentioned in the submitted article.

\section{References}

1. Eugui EM, Almquist SJ, Muller CD, Allison AC. Lymphocyte-selective cytostatic and immunosuppressive effects of mycophenolic acid in vitro: role of deoxyguanosine nucleotide depletion. Scand J Immunol. 1991;33(2):161-173.

2. Gosio B. Sperimentate su culture pure di bacilli del carbpnchio demostrarano notevole potere antisettica. G Accad Med Torino. 1983;61:484.

3. Franklin TJ, Cook JM. The inhibition of nucleic acid synthesis by mycophenolic acid. Biochem J. 1969;113(3):515-524.

4. Morris RE, Wang J, Blum JR, et al. Immunosuppressive effects of the morpholinoethyl ester of mycophenolic acid (RS-61443) in rat and nonhuman primate recipients of heart allografts. Transplant Proc. 1991; Apr 23(2 Suppl 2):19-25.

5. Platz KP, Bechstein WO, Eckhoff DE, Suzuki Y, Sollinger HW RS-61443 reverses acute allograft rejection in dogs. Surgery. 1991;110(4):736-740; discussion 40-41.

6. Sollinger HW, Deierhoi MH, Belzer FO, Diethelm AG, Kauffman RS RS-61443-a phase I clinical trial and pilot rescue study. Transplantation. 1992;53(2):428-432.

7. Carr SF, Papp E, Wu JC, Natsumeda Y. Characterization of human type I and type II IMP dehydrogenases. J Biol Chem. 1993;268(36):27286-27290.

8. Allison AC, Eugui EM. Mycophenolate mofetil and its mechanisms of action. Immunopharmacology. 2000;47(2-3):85-118.

9. Huang HD, Liu ZH, Zhu XJ, Chen ZH, Li LS. Inhibition of mycophenolic acid on NF-kappaB activity in human endothelial cells. Acta pharmacol Sin. 2002;23(7):649-653.

10. Laurent AF, Dumont S, Poindron P, Muller CD. Mycophenolic acid suppresses protein $\mathrm{N}$-linked glycosylation in human monocytes and their adhesion to endothelial cells and to some substrates. Exp Hematol. 1996;24(1):59-67.

11. Raab M, Daxecker H, Karimi A, et al. In vitro effects of mycophenolic acid on the nucleotide pool and on the expression of adhesion molecules of human umbilical vein endothelial cells. Clin Chim Acta. 2001;310(1):89-98.

12. Allison AC, Eugui EM. Mechanisms of action of mycophenolate mofetil in preventing acute and chronic allograft rejection. Transplantation. 2005;80(2 Suppl):S181-S190.

13. Bullingham R, Monroe S, Nicholls A, Hale M. Pharmacokinetics and bioavailability of mycophenolate mofetil in healthy subjects after single-dose oral and intravenous administration. Journal of Clinical Pharmacology. 1996;36(4):315-324.

14. Sweeney MJ, Hoffman DH, Esterman MA. Metabolism and biochemistry of mycophenolic acid. Cancer Res. 1972;32(9):1803-1809.

15. Staatz CE, Tett SE. Clinical pharmacokinetics and pharmacodynamics of mycophenolate in solid organ transplant recipients. Clin Pharmacokinet. 2007;46(1):13-58.

16. Shaw LM, Mick R, Nowak I, Korecka M, Brayman KL. Pharmacokinetics of mycophenolic acid in renal transplant patients with delayed graft function. J Clin Pharmacol. 1998;38(3):268-275.

17. Shaw LM, Nawrocki A, Korecka M, Solari S, Kang J. Using established immunosuppressant therapy effectively: lessons from the measurement of mycophenolic acid plasma concentrations. Ther Drug Monit. 2004;26(4):347-351.

18. Weber LT, Shipkova M, Lamersdorf T, et al. Pharmacokinetics of mycophenolic acid (MPA) and determinants of MPA free fraction in pediatric and adult renal transplant recipients. German Study group on Mycophenolate Mofetil Therapy in Pediatric Renal Transplant Recipients. J Am Soc Nephrol. 1998;9(8):1511-1520. 
19. HaleMD, Nicholls AJ, Bullingham RE, et al. The pharmacokinetic-pharmacodynamic relationship for mycophenolate mofetil in renal transplantation. Clin Pharmacol Ther. 1998;64(6):672-683.

20. Kiberd BA, Lawen J, Fraser AD, Keough-Ryan T, Belitsky P. Early adequate mycophenolic acid exposure is associated with less rejection in kidney transplantation. Am J Transplant. 2004;4(7):1079-1083.

21. van Gelder T, Le Meur Y, Shaw LM, et al. Therapeutic drug monitoring of mycophenolate mofetil in transplantation. Ther Drug Monit. 2006;28(2):145-154.

22. Filler G, Mai I. Limited sampling strategy for mycophenolic acid area under the curve. Ther Drug Monit. 2000;22(2):169-173.

23. Pawinski T, Hale M, Korecka M, Fitzsimmons WE, Shaw LM. Limited sampling strategy for the estimation of mycophenolic acid area under the curve in adult renal transplant patients treated with concomitant tacrolimus. Clin Chem. 2002;48(9):1497-1504.

24. Placebo-controlled study of mycophenolate mofetil combined with cyclosporin and corticosteroids for prevention of acute rejection. European Mycophenolate Mofetil Cooperative Study Group. Lancet. 1995;345(8961):1321-1325.

25. A blinded, randomized clinical trial of mycophenolate mofetil for the prevention of acute rejection in cadaveric renal transplantation. The Tricontinental Mycophenolate Mofetil Renal Transplantation Study Group. Transplantation. 1996;61(7):1029-1037.

26. Sollinger HW. Mycophenolate mofetil for the prevention of acute rejection in primary cadaveric renal allograft recipients. US Renal Transplant Mycophenolate Mofetil Study Group. Transplantation. 1995;60(3):225-232.

27. Halloran P, Mathew T, Tomlanovich S, Groth C, Hooftman L, Barker C. Mycophenolate mofetil in renal allograft recipients: a pooled efficacy analysis of three randomized, double-blind, clinical studies in prevention of rejection. The International Mycophenolate Mofetil Renal Transplant Study Groups. Transplantation. 1997;63(1):39-47.

28. Remuzzi G, Lesti M, Gotti E, et al. Mycophenolate mofetil versus azathioprine for prevention of acute rejection in renal transplantation (MYSS): a randomised trial. Lancet. 2004;364(9433):503-512.

29. Remuzzi G, Cravedi P, Costantini M, et al. Mycophenolate mofetil versus azathioprine for prevention of chronic allograft dysfunction in renal transplantation: the MYSS follow-up randomized, controlled clinical trial. J Am Soc Nephrol. 2007;18(6):1973-1985.

30. Mathew TH. A blinded, long-term, randomized multicenter study of mycophenolate mofetil in cadaveric renal transplantation: results at three years. Tricontinental Mycophenolate Mofetil Renal Transplantation Study Group. Transplantation. 1998;65(11):1450-1454.

31. Ojo AO, Meier-Kriesche HU, Hanson JA, et al. Mycophenolate mofetil reduces late renal allograft loss independent of acute rejection. Transplantation. 2000;69(11):2405-2409.

32. Meier-Kriesche HU, Steffen BJ, Hochberg AM, et al. Mycophenolate mofetil versus azathioprine therapy is associated with a significant protection against long-term renal allograft function deterioration. Transplantation. 2003;75(8):1341-1346.

33. Meier-Kriesche HU, Steffen BJ, Hochberg AM, et al. Long-term use of mycophenolate mofetil is associated with a reduction in the incidence and risk of late rejection. Am J Transplant. 2003;3(1):68-73.

34. Mycophenolate mofetil for the treatment of refractory, acute, cellular renal transplant rejection. The Mycophenolate Mofetil Renal Refractory Rejection Study Group. Transplantation. 1996;61(5):722-729.

35. Mycophenolate mofetil for the treatment of a first acute renal allograft rejection: The Mycophenolate Mofetil Acute Renal Rejection Study Group. Transplantation. 1998;65(2):235-241.

36. Flechner SM. Minimizing calcineurin inhibitor drugs in renal transplantation. Transplant Proc. 2003;35(3 Supp1):S118-S121.

37. Calne RY, White DJ, Thiru S, et al. Cyclosporin A in patients receiving renal allografts from cadaver donors. Lancet. 1978;2(8104-5):1323-1327.

38. Hariharan S, Johnson CP, Bresnahan BA, Taranto SE, McIntosh MJ, Stablein D. Improved graft survival after renal transplantation in the United States, 1988 to 1996. N Engl J Med. 2000;342(9):605-612.
39. Curtis JJ, Luke RG, Dubovsky E, Diethelm AG, Whelchel JD, Jones P. Cyclosporin in therapeutic doses increases renal allograft vascular resistance. Lancet. 1986;2(8505):477-479.

40. Keogh A. Calcineurin inhibitors in heart transplantation. J Heart Lung Transplant. 2004;23(5 Suppl):S202-S206.

41. Sanders CE, Jr., Curtis JJ. Role of hypertension in chronic renal allograft dysfunction. Kidney Int. 1995;52:S43-S47.

42. Smak Gregoor PJ, van Gelder T, van Besouw NM, van der Mast BJ, JN IJ, Weimar W. Randomized study on the conversion of treatment with cyclosporine to azathioprine or mycophenolate mofetil followed by dose reduction. Transplantation. 2000;70(1):143-148.

43. Abramowicz D, Manas D, Lao M, et al. Cyclosporine withdrawal from a mycophenolate mofetil-containing immunosuppressive regimen in stable kidney transplant recipients: a randomized, controlled study. Transplantation. 2002;74(12):1725-1734.

44. Schnuelle $\mathrm{P}$, van der Heide JH, Tegzess A, et al. Open randomized trial comparing early withdrawal of either cyclosporine or mycophenolate mofetil in stable renal transplant recipients initially treated with a triple drug regimen. J Am Soc Nephrol. 2002;13(2):536-543.

45. Dudley C, Pohanka E, Riad H, et al. Mycophenolate mofetil substitution for cyclosporine a in renal transplant recipients with chronic progressive allograft dysfunction: the "creeping creatinine" study. Transplantation. 2005;79(4):466-475

46. Flechner SM, Goldfarb D, Modlin C, et al. Kidney transplantation without calcineurin inhibitor drugs: a prospective, randomized trial of sirolimus versus cyclosporine. Transplantation. 2002;74(8):1070-1076.

47. Flechner SM, Kurian SM, Solez K, et al. De novo kidney transplantation without use of calcineurin inhibitors preserves renal structure and function at two years. Am J Transplant. 2004;4(11):1776-1785.

48. Grinyo JM, Gil-Vernet S, Cruzado JM, et al. Calcineurin inhibitor-free immunosuppression based on antithymocyte globulin and mycophenolate mofetil in cadaveric kidney transplantation: results after 5 years. Transpl Int. 2003;16(11):820-827.

49. Kreis H, Cisterne JM, Land W, et al. Sirolimus in association with mycophenolate mofetil induction for the prevention of acute graft rejection in renal allograft recipients. Transplantation. 2000;69(7):1252-1260.

50. Lo A, Egidi MF, Gaber LW, et al. Comparison of sirolimus-based calcineurin inhibitor-sparing and calcineurin inhibitor-free regimens in cadaveric renal transplantation. Transplantation. 2004;77(8):1228-1235.

51. Stegall MD, Larson TS, Prieto M, et al. Kidney transplantation without calcineurin inhibitors using sirolimus. Transplant Proc. 2003;35(3 Suppl):S125-S127.

52. Theodorakis J, Schneeberger H, Illner WD, Stangl M, Zanker B, Land W. Nephrotoxicity-free, mycophenolate mofetil-based induction/maintenance immunosuppression in elderly recipients of renal allografts from elderly cadaveric donors. Transplantation proceedings. 2000;32(1A Supp1):S9-S11.

53. Tran HT, Acharya MK, McKay DB, et al. Avoidance of cyclosporine in renal transplantation: effects of daclizumab, mycophenolate mofetil, and steroids. J Am Soc Nephrol. 2000;11(10):1903-1909.

54. Vincenti F, Ramos E, Brattstrom C, et al. Multicenter trial exploring calcineurin inhibitors avoidance in renal transplantation. Transplantation. 2001;71(9):1282-1287.

55. Larson TS, Dean PG, Stegall MD, et al. Complete avoidance of calcineurin inhibitors in renal transplantation: a randomized trial comparing sirolimus and tacrolimus. Am J Transplant. 2006;6(3):514-522.

56. Flechner SM, Goldfarb D, Solez K, et al. Kidney transplantation with sirolimus and mycophenolate mofetil-based immunosuppression: 5-year results of a randomized prospective trial compared to calcineurin inhibitor drugs. Transplantation. 2007;83(7):883-892.

57. Ekberg H, Tedesco-Silva H, Demirbas A, et al. Reduced exposure to calcineurin inhibitors in renal transplantation. $N$ Engl $\mathrm{J} \mathrm{Med}$. 2007;357(25):2562-2575.

58. Srinivas TR, Schold JD, Guerra G, Eagan A, Bucci CM, Meier-Kriesche HU. Mycophenolate mofetil/sirolimus compared to other common immunosuppressive regimens in kidney transplantation. $\mathrm{Am} \mathrm{J}$ Transplant. 2007;7(3):586-594. 
59. Veenstra DL, Best JH, Hornberger J, Sullivan SD, Hricik DE. Incidence and long-term cost of steroid-related side effects after renal transplantation. Am J Kidney Dis. 1999;33(5):829-839.

60. Montagnino G, Tarantino A, Maccario M, Elli A, Cesana B, Ponticelli C. Long-term results with cyclosporine monotherapy in renal transplant patients: a multivariate analysis of risk factors. Am J Kidney Dis. 2000;35(6):1135-1143.

61. Parrott NR, Hammad AQ, Watson CJ, Lodge JP, Andrews CD. Multicenter, randomized study of the effectiveness of basiliximab in avoiding addition of steroids to cyclosporine a monotherapy in renal transplant recipients. Transplantation. 2005;79(3):344-348.

62. Hricik DE, Whalen CC, Lautman J, et al. Withdrawal of steroids after renal transplantation-clinical predictors of outcome. Transplantation. 1992;53(1):41-45.

63. Hricik DE, O’Toole MA, Schulak JA, Herson J. Steroid-free immunosuppression in cyclosporine-treated renal transplant recipients: a meta-analysis. J Am Soc Nephrol. 1993;4(6):1300-1305.

64. Kasiske BL, Chakkera HA, Louis TA, Ma JZ. A meta-analysis of immunosuppression withdrawal trials in renal transplantation. $J \mathrm{Am}$ Soc Nephrol. 2000;11(10):1910-1917.

65. Squifflet JP, Vanrenterghem Y, van Hooff JP, Salmela K, Rigotti P. Safe withdrawal of corticosteroids or mycophenolate mofetil: results of a large, prospective, multicenter, randomized study. Transplantation proceedings. 2002;34(5):1584-1586.

66. Vanrenterghem Y, Lebranchu Y, Hene R, Oppenheimer F, Ekberg H. Double-blind comparison of two corticosteroid regimens plus mycophenolate mofetil and cyclosporine for prevention of acute renal allograft rejection. Transplantation. 2000;70(9):1352-1359.

67. Matas AJ, Ramcharan T, Paraskevas S, et al. Rapid discontinuation of steroids in living donor kidney transplantation: a pilot study. Am J Transplant. 2001;1(3):278-283.

68. Matas AJ, Kandaswamy R, Humar A, et al. Long-term immunosuppression, without maintenance prednisone, after kidney transplantation. Annals of surgery. 2004;240(3):510-516; discussion 6-7.

69. Vincenti F, Monaco A, Grinyo J, Kinkhabwala M, Roza A. Multicenter randomized prospective trial of steroid withdrawal in renal transplant recipients receiving basiliximab, cyclosporine microemulsion and mycophenolate mofetil. Am J Transplant. 2003;3(3):306-311.

70. Vincenti F, Schena FP, Paraskevas S, Hauser IA, Walker RG, Grinyo J. A randomized, multicenter study of steroid avoidance, early steroid withdrawal or standard steroid therapy in kidney transplant recipients. Am J Transplant. 2008;8(2):307-316.

71. ter Meulen CG, van Riemsdijk I, Hene RJ, et al. Steroid-withdrawal at 3 days after renal transplantation with anti-IL-2 receptor alpha therapy: a prospective, randomized, multicenter study. Am J Transplant. 2004;4(5):803-810.

72. Gallon LG, Winoto J, Leventhal JR, Parker MA, Kaufman DB. Effect of prednisone versus no prednisone as part of maintenance immunosuppression on long-term renal transplant function. Clin J Am Soc Nephrol. 2006;1(5):1029-1038.

73. Pascual J, Quereda C, Zamora J, Hernandez D. Updated metaanalysis of steroid withdrawal in renal transplant patients on calcineurin inhibitor and mycophenolate mofetil. Transplant Proc. 2005;37(9):3746-3748.
74. Hardinger KL, Brennan DC, Lowell J, Schnitzler MA. Long-term outcome of gastrointestinal complications in renal transplant patients treated with mycophenolate mofetil. Transpl Int. 2004;17(10):609-616.

75. Pelletier RP, Akin B, Henry ML, et al. The impact of mycophenolate mofetil dosing patterns on clinical outcome after renal transplantation. Clin Transplant. 2003;17(3):200-205.

76. Tierce JC, Porterfield-Baxa J, Petrilla AA, Kilburg A, Ferguson RM. Impact of mycophenolate mofetil (MMF)-related gastrointestinal complications and MMF dose alterations on transplant outcomes and healthcare costs in renal transplant recipients. Clin Transplant. 2005;19(6):779-784.

77. Knoll GA, MacDonald I, Khan A, Van Walraven C. Mycophenolate mofetil dose reduction and the risk of acute rejection after renal transplantation. J Am Soc Nephrol. 2003;14(9):2381-2386.

78. Wang K, Zhang H, Li Y, et al. Safety of mycophenolate mofetil versus azathioprine in renal transplantation: a systematic review. Transplant Proc. 2004;36(7):2068-2070.

79. Chan L, Mulgaonkar S, Walker R, Arns W, Ambuhl P, Schiavelli R. Patient-reported gastrointestinal symptom burden and health-related quality of life following conversion from mycophenolate mofetil to enteric-coated mycophenolate sodium. Transplantation. 2006;81(9):1290-1297.

80. Bunnapradist S, Lentine KL, Burroughs TE, et al. Mycophenolate mofetil dose reductions and discontinuations after gastrointestinal complications are associated with renal transplant graft failure. Transplantation. 2006;82(1):102-107.

81. Budde K, Bauer S, Hambach P, et al. Pharmacokinetic and pharmacodynamic comparison of enteric-coated mycophenolate sodium and mycophenolate mofetil in maintenance renal transplant patients. Am J Transplant. 2007;7(4):888-898.

82. Salvadori M, Holzer H, de Mattos A, et al. Enteric-coated mycophenolate sodium is therapeutically equivalent to mycophenolate mofetil in de novo renal transplant patients. Am J Transplant. 2004;4(2):231-236.

83. Salvadori M, Holzer H, Civati G, et al. Long-term administration of enteric-coated mycophenolate sodium (EC-MPS; myfortic) is safe in kidney transplant patients. Clinical nephrology. 2006;66(2):112-119.

84. Budde K, Glander P, Diekmann F, et al. Enteric-coated mycophenolate sodium: safe conversion from mycophenolate mofetil in maintenance renal transplant recipients. Transplant Proc. 2004;36(2 Suppl):S524-S527.

85. Budde K, Knoll G, Curtis J, et al. Safety and efficacy after conversion from mycophenolate mofetil to enteric-coated mycophenolate sodium: results of a 1-year extension study. Transplant Proc. 2005;37(2):912-915.

86. Legendre C, Cohen D, Zeier M, Rostaing L, Budde K. Efficacy and safety of enteric-coated mycophenolate sodium in de novo renal transplant recipients: pooled data from three 12-month multicenter, openlabel, prospective studies. Transplant Proc. 2007;39(5):1386-1391.

87. Pietruck F, Abbud-Filho M, Vathsala A, Massari PU, Po-Huang L, Nashan B. Conversion from mycophenolate mofetil to enteric-coated mycophenolate sodium in stable maintenance renal transplant patients: pooled results from three international, multicenter studies. Transplant Proc. 2007;39(1):103-108. 
